\title{
NUEVOS FACTORES QUE INCIDEN SOBRE LA CALIDAD DE LA EDUCACIÓN MÉDICA Y DE LAS PROFESIONES DE SALUD
}

\author{
NEW FACTORS AFFECTING THE QUALITY OF MEDICAL EDUCATION AND HEALTH \\ PROFESSIONS
}

\author{
Graciela Risco de Domínguez,a
}

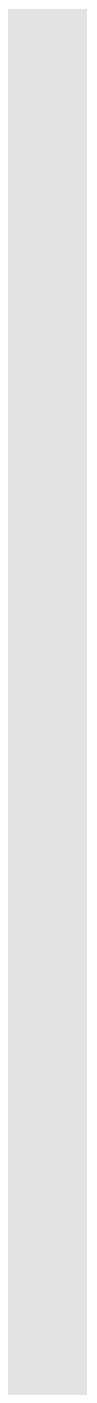

\begin{abstract}
Las universidades peruanas deben demostrar que cumplen con condiciones básicas de calidad (CBC) para obtener la licencia institucional que les permite seguir ofreciendo el servicio educativo universitario. El licenciamiento institucional es obligatorio y es conducido por la Superintendencia Nacional de Educación Superior Universitaria (SUNEDU), creada por la Ley Universitaria N $30220^{(1)}$. Entre las atribuciones de la SUNEDU están: aprobar o denegar las solicitudes de licenciamiento de universidades, filiales, escuelas y programas de estudio.
\end{abstract}

En diciembre de 2015, fecha de inicio de las funciones de la SUNEDU, existían 143 universidades, en febrero de 2019 habían recibido la licencia de funcionamiento 62 de ellas. La licencia de funcionamiento fue denegada a seis universidades que no podrán seguir funcionando, las 75 restantes están en proceso de licenciamiento ${ }^{(2)}$.

Dentro del proceso de licenciamiento institucional, la SUNEDU se prepara para iniciar el licenciamiento de programas de pregrado de Medicina (PPM) por las siguientes razones principales ${ }^{(3)}$ :

a. El alto impacto de la calidad de la educación médica sobre la salud y el bienestar de la población.

b. El marcado incremento del número de facultades o escuelas de medicina a partir de 2007, las que no aseguran una formación profesional de calidad.

No es la primera vez que en Perú se establece un sistema de evaluación de la calidad de la educación médica, en julio de 1999 entra en vigencia la Ley № $271544^{(4)}$ que establece la acreditación obligatoria de las facultades o escuelas de Medicina y suspende la creación de nuevas facultades o escuelas. La Ley crea la Comisión para la Acreditación de Facultades o Escuelas de Medicina (CAFME). Las razones para la creación de CAFME fueron mejorar la calidad y detener el incremento del número de escuelas de medicina. La CAFME creó estándares mínimos para la acreditación de facultades o escuelas de medicina y para la evaluación previa a la creación de nuevas facultades. La CAFME estuvo vigente hasta el 2007, en el periodo de su vigencia acreditó a 23 de las 28 facultades de medicina existentes, las facultades no acreditadas continuaron su funcionamiento ya que CAFME no tenía atribuciones para cerrarlas.

En lo académico, la CAFME introdujo la cultura de autoevaluación y acreditación de la calidad educativa; e introdujo el modelo de educación por competencias en la educación médica. Su logro más reconocido fue impedir la creación de nuevas facultades de medicina, sólo autorizó el funcionamiento de una nueva escuela, la cual diseñó e implementó un currículo por competencias, como los prescribía la CAFME, e introdujo innovaciones metodológicas de importancia para la formación de médicos en el país ${ }^{{ }^{(5)}}$. Al dejar de funcionar la CAFME, la evaluación de la calidad de los programas de medicina y la creación de nuevas escuelas de medicina dejó de estar regulada ocasionando que el número de PPM se incrementara, de 29 en 2007 a 51 en 2019, no estando garantizada su calidad.

\footnotetext{
Universidad Tecnológica del Perú. Lima, Perú.

Médico cirujano, doctora en Medicina.

Recibido 06/03/2019 Aprobado: 06/03/2019 En línea 15/03/2019
}

Citar como: Risco de Domínguez G. Nuevos factores que inciden sobre la calidad de la educación médica y de las profesiones de salud. Rev Peru Med Exp Salud Publica. 2019;36(1):7-9.doi:10.17843/rpmesp.2019.361.4337. 
Próximos a reiniciar el proceso de evaluación de la calidad de los PPM después de 12 años de su desactivación, resulta necesario analizar los nuevos retos que impulsan la transformación de la atención de salud y de la educación de los médicos y de todos los profesionales de la salud, que deberían dar lugar a nuevos indicadores de calidad de la educación en salud. Señalamos los que consideramos más importantes:

1. El Consenso Global sobre la Responsabilidad Social de las escuelas de medicina de formar profesionales competentes para contribuir a mejorar los sistemas de salud y la situación de salud de la población que atienden ${ }^{(6)}$ y el Consenso para formar líderes competentes capaces de transformar los sistemas de salud ${ }^{(7)}$. Los consensos mencionados comprometen a las escuelas de medicina a alinear las competencias del perfil profesional del médico a las necesidades de salud de la población y de los sistemas de salud que atienden.

2. Lograr el acceso de todas las personas a servicios de salud esenciales y de calidad sin pasar apuros económicos ${ }^{(8)}$. La atención primaria de la salud (APS) es la principal estrategia para lograr el acceso universal a la salud. En el Perú se trata de implantar la APS mediante la conformación de Redes Integradas de Salud ${ }^{(9)}$, un factor importante para lograrlo es contar con profesionales que tengan la motivación y las competencias adecuadas para ello. Con este propósito se debería incluir en el currículo actividades formativas en el primer nivel de atención y no sólo en hospitales de segundo o tercer nivel. Se requiere además desarrollar en el estudiante competencias para la prevención y promoción de la salud. Lo dicho se aplica también para enfermeras, obstetras y otros profesionales de la salud, quienes tienen un rol fundamental en el equipo de salud.

3. Transformar los servicios de salud para que brinden un trato humano y ético a las personas. Como respuesta a la «deshumanización» de la medicina surge el movimiento internacional que impulsa la Medicina Centrada en la Persona ${ }^{(10)}$. Para lograrlo se requiere formar a los profesionales de la salud para que conciban a la persona integralmente en sus aspectos biológicos, psicológicos y sociales, y que a las competencias de formación científica se acompañen competencias de formación personal como comunicación, empatía, compasión y ética.

4. Brindar atención médica de calidad a la población mayor de 60 años. El envejecimiento de la población es un fenómeno mundial, en el Perú según datos del Instituto Nacional de Estadística e Informática, se calcula que en el 2050 el $21 \%$ de la población peruana serán personas mayores de 60 años. El envejecimiento de la población trae consigo una carga de enfermedades crónicas no trasmisibles que requiere que el personal de salud esté preparado para brindar atención continua en ambientes hospitalarios o en el hogar. Además, requiere la capacidad de trabajar en equipos interprofesionales, conociendo y valorando las competencias de los miembros del equipo.

5. Formar profesionales capaces de aprender durante toda la vida. La abundancia de los conocimientos científicos y su rápida renovación hace necesario que la facultad dosifique los conocimientos impartidos e integre los conocimientos científicos básicos a la formación clínica. Implica también que la facultad debe sustituir el modelo educativo tradicional centrado en el profesor y en la trasmisión de conocimientos por un modelo educativo por competencias que forme a los profesionales con conocimientos, habilidades y actitudes para la gestión de la información y para su análisis crítico y reflexivo y que estén motivados para aprender en forma autónoma durante toda la vida.

6. Manejo efectivo y ético de las tecnologías de la información y las comunicaciones (TIC) ${ }^{(11)}$. El gran desarrollo de las TIC está transformando todos los ámbitos de la vida, en especial la salud y la educación. Internet permite el acceso a la información en salud, la telemedicina permite mejorar la salud de poblaciones alejadas, la historia clínica electrónica se difunde cada vez más, la creación de dispositivos electrónicos (wearables) que colocados en el cuerpo trasmiten datos biológicos a distancia, los robots que asisten a las personas mayores, el desarrollo de la inteligencia artificial capaz de procesar enormes cantidades de información, todo esto relacionado con la transformación digital de la salud. Los profesionales de la salud deben estar preparados para usar la tecnología en beneficio de sus pacientes y en apoyo de su actividad profesional. Las universidades deberían incluir en el proceso de enseñanza-aprendizaje la tecnología digital que mejora el aprendizaje y familiariza al estudiante con su uso y aplicación adecuada, especialmente cuando en la actualidad los estudiantes universitarios son nativos digitales y usan varios dispositivos y aplicaciones digitales.

7. La tecnología transforma los métodos clásicos de educación en salud. Por ejemplo, ya no se necesita disecar cadáveres para aprender anatomía, el uso de maquetas y software permite una visualización en dos y tres planos de las estructuras anatómicas y sustituye con ventaja el uso de cadáveres ${ }^{(12)}$. Por lo tanto, no se requiere contar con un anfiteatro anatómico ni con cadáveres, evitando así altos costos y los efectos dañinos del formol. Otro ejemplo, lo constituye la adquisición de las competencias de formación personal y clínica, las que pueden aprenderse usando pacientes simulados, pacientes virtuales o robots. La simulación ha mostrado un gran avance en los últimos diez años, se han estandarizado los procesos y se han realizado 
estudios sistemáticos que demuestran que se puede reemplazar un porcentaje de horas clínicas por horas de prácticas en el centro de simulación ${ }^{(13,14)}$, lo cual puede conducir a disminuir la sobresaturación de los campos clínicos. Adicionalmente, la simulación tiene la ventaja de preparar al estudiante en habilidades clínicas antes de intervenir sobre un paciente, evitando el daño y mejorando la seguridad del paciente.

El sistema de aseguramiento de la calidad ${ }^{(15)}$ de la educación superior universitaria peruana establece que el licenciamiento y la acreditación son procesos sucesivos, distintos pero complementarios. En el caso de los PPM, ambos son procesos obligatorios y deben estar bien articulados para garantizar la calidad de la educación médica, sin obstaculizar su innovación.
Los retos aquí planteados corresponden a tendencias globales que se manifiestan con características propias en la realidad peruana, son retos que irán profundizándose en el transcurso del siglo XXI. Frente a ellos, las entidades educativas deberían formar profesionales preparados para los cambios que las condiciones del ejercicio profesional les impondrán, y contribuir a la mejora de las condiciones de salud de la población peruana. Debemos tener en cuenta que la formación de un profesional de salud toma entre cinco a siete años y que ejercerá su profesión por unos 40 años después del egreso, es decir que estamos formando profesionales, por lo menos hasta el 2070. Se hace evidente que el diseño curricular de calidad debe tomar en cuenta las necesidades actuales de salud y sus proyecciones futuras.

\section{REFERENCIAS BIBLIOGRÁFICAS}

1. Ley Universitaria. Ley $\mathrm{N}^{\circ}$ 30220. El Peruano, No 12914, (9 julio 2014). Disponible en: https://www.sunedu.gob. pe/wp-content/uploads/2017/04/Leyuniversitaria-30220.pdf

2. Superintendencia Nacional de Educación Superior Universitaria [Internet]. Lima: SUNEDU; 2018 [Consultado el 26 de febrero 2019]. Avances y estatus del Licenciamiento. Disponible en: https://www.sunedu.gob.pe/avanceslicenciamiento/

3. Superintendencia Nacional de Educación Superior Universitaria [Internet]. Lima: SUNEDU; 2018 [Consultado el 26 de febrero 2019]. Proceso del Licenciamiento. Disponible en: https://www.sunedu. gob.pe/direccion-de-licenciamientofunciones/

4. Ley que institucionaliza la Acreditación de Facultades o Escuelas de Medicina. Ley ${ }^{\circ}$ 27154. El Peruano (11 de julio de 1999). Disponible en: https://docs.peru.justia. com/federales/leyes/27154-jul-7-1999.pdf

5. Risco de Domínguez G. Diseño e implementación de un currículo por competencias para la formación de médicos. Rev Per Med Exp Salud Publica. 2014;31(3):572-81.

6. Boelem C, Woolard R. Consenso global sobre la responsabilidad social de las facultades de medicina. Educ Med 2011;14(1):7-14.
7. Frenk J, Chen L, Bhutta ZA, Cohen J, Crisp N, Evans T, et al. Health professionals for a new century: transforming education to strengthen health systems in an interdependent world. Lancet. 2010;376(9756):1923-58. doi: 10.1016/S0140-6736(10)61854-5.

8. World Health Organization [Internet]. Geneva: WHO; 2019 [Consultado el 26 de febrero 2019]. WHO priorities. Disponible en: https://www.who.int/dg/ priorities/en/

9. Lineamientos parala Prestación de Servicios de Salud en las Redes Integradas de Salud (Redes Integradas de Atención Primaria de la Salud). RM N ${ }^{\circ}$ 167-2017. Lima: Ministerio de Salud: 2017. Disponible en: $\mathrm{ftp}: / / \mathrm{ftp} 2 . \mathrm{minsa} . g o b . p e /$ descargas/dgain/ web/normas/CD\%20NORMAS\%20 $\% 20$ TEMATICAS/REDES/RM\%20 LINEAMIENTOS\%20REDES\%20\%20 167-2017-SA.pdf

10. Mezzich JE, Perales A. Atención Clínica Centrada en la Persona: principios y estrategias. Rev Per Med Exp Salud Publica. 2016;33(4):794-800. doi: 10.17843/ rpmesp.2016.334.2567.

11. World Federation for Medical Education. Basic Medical Education WFME Global Standards for Quality Improvement. The 2015 Revision. Copenhagen: WFME; 2015. Disponible en: https://wfme.org/ publications/wfme-global-standards-for- quality-improvement-bme/?wpdmdl=83 1\&ind=kjMLv1eFyMHOlAmSx3U3T Oh-pf9f2FwGErfIn8ZqFhM1kNl6m5_ No-EUbWgcmenN

12. Losco CD, Grant WD, Armson A, Meyer AJ,WalkerBF.Effectivemethodsofteaching and learning in anatomy as a basic science. A BEME systematic review: BEME guide no. 44. Med Teach. 2017;39(3):234-243. doi: 10.1080/0142159X.2016.1271944.

13. Motola I, Devine LA, Chung HS, Sullivan JE, Issenberg SB. Simulation in healthcare education: a best evidence practical guide. AMEE Guide No. 82. Med Teach. 2013;35(10):e1511-30. doi: 10.3109/0142159X.2013.818632.

14. Hayden JK, Smiley RA, Alexander M, Kardong-Edgren S, Jeffries PR. The NCSBN National Simulation Study: A Longitudinal, Randomized, Controlled Study Replacing Clinical Hours with Simulation in Prelicensure Nursing Education.J Nurs Regul.2014;5(2):C1-S64. doi: 10.1016/S2155-8256(15)30061-2.

15. Política para el Aseguramiento de la Calidad de la Educación Superior Universitaria Ministerio de Educación DS N 016-2015-MINEDU. El Peruano, No562355 (26 de septiembre de 2015). Disponible en: https://www.sunedu.gob. pe/aprueban-la-politica-de-aseguramientode-la-calidad-de-la-educacion-superioruniversitaria/ 